\title{
Visions: The Coming Revolutions in Particle Physics
}

\author{
Chris Quigg \\ Fermi National Accelerator Laboratory, ${ }^{*}$ P.O. Box 500, Batavia, Illinois 60510 USA; \\ e-mail: quigg@fnal.gov
}

\begin{abstract}
Wonderful opportunities await particle physics over the next decade, with the coming of the Large Hadron Collider to explore the 1-TeV scale (extending efforts at LEP and the Tevatron to unravel the nature of electroweak symmetry breaking) and many initiatives to develop our understanding of the problem of identity and the dimensionality of spacetime.
\end{abstract}

PACS: 12.15.-y 13.85.-t 12.60.-i 14.80.Bn

FERMILAB-CONF-02/058-T

\section{Galileo's Three Revolutions}

In this talk, I want to evoke some of the revolutionary developments I believe will come over the next two decades in particle physics. To set the context, and because we find ourselves in Italy, I'd like to begin by recalling three revolutions we identify with Galileo and his time.

Eppur si muove. In the public mind and in popular literature, Galileo is remembered chiefly for his part in completing the Copernican revolution, establishing that humans do not occupy a privileged location in the universe. It's a great achievement and a good story, given texture by Galileo's complex persona and by the richness of his relationship with the inquistorial Church [1].

Cimenti. The Copernican revolution is a scientific movement accomplished. We scientists revere Galileo no less for his contribution to the scientific method. For it was during Galileo's time that humans found the courage to reject Authority. They learned instead to read nature by doing experiments, subjecting their hypotheses to unremitting trials by ordeal that Galileo called cimenti. The notion that experiment, not eloquence, is the arbiter of what is true revolutionized mankind's relationship with nature.

The minute particular. An essential element of civilization is human curiosity about the world and a thirst to comprehend nature. Until five centuries ago, the questions our ancestors wondered about were broad and the explanations they advanced were sweeping but vague. Asking great questions - seeking to explain everything about the world all at once - led to extremely limited answers. Science as we know it took shape in Galileo's time when humans learned that asking limited questions could lead them to universal insights. In contemporary

\footnotetext{
*Fermilab is operated by Universities Research Association Inc. under Contract No. DEAC02-76CH03000 with the United States Department of Energy.
} 
American discourse at least, the received wisdom holds that all the great questions have been answered and that today's scientists - gifted though they might be - are dealing with ever narrower research topics. This canard betrays an ignorance of how science has been done ever since it became worthy of the name. You can help explain to the world what science is. To be most effective, you must make the connection between your own minute particular (the search for the Higgs boson, the width of the $W$, the mass of the top, or whatever) and the universal understanding we are trying to build. .

Are all the great scientific revolutions in the distant past? Not at all: We are here to discuss the revolution-in-progress that we expect experiments with the Large Hadron Collider to complete. This is the radically new and simple conception of matter brought about by the development of gauge theories and the recognition that quarks and leptons are the basic constituents of matterat the current limits of our resolution. The gauge-theory synthesis is part of a larger change that we are living through - no, making - in the way humans think about their world. The recognition that the human scale is not privileged, that we need to leave our familiar surroundings the better to understand them, has been building since the birth of quantum mechanics. As it emerges whole, fully formed, in our unified theories and renormalization group equations, the notion seems to me both profound and irresistible. I find it fully appropriate to compare this change in perception with the shifts in viewpoint we owe to Copernicus and Einstein.

\section{Our Picture of Matter}

We base our understanding of physical phenomena on the identification of a few constituents that seem elementary at the current limits of resolution of about $10^{-18} \mathrm{~m}$, and a few fundamental forces. The constituents are the pointlike quarks $\left\{(u, d)_{L},(c, s)_{L},(t, b)_{L}\right\}$ and leptons $\left\{\left(\nu_{e}, e\right)_{L},\left(\nu_{\mu}, \mu\right)_{L},\left(\nu_{\tau}, \tau\right)_{L}\right\}$, with strong, weak, and electromagnetic interactions specified by $S U(3)_{c} \otimes S U(2)_{L} \otimes U(1)_{Y}$ gauge symmetries.

The electroweak theory is founded on the weak-isospin symmetry embodied in the quark and lepton doublets and weak-hypercharge phase symmetry, plus the idealization that neutrinos are massless. [I In its simplest form, with the electroweak gauge symmetry broken by the Higgs mechanism, the $S U(2)_{L} \otimes U(1)_{Y}$ theory has scored many qualitative successes: the prediction of neutral-current interactions, the necessity of charm, the prediction of the existence and properties of the weak bosons $W^{ \pm}$and $Z^{0}$. Over the past ten years, in great measure due to the beautiful experiments carried out at the $Z$ factories at CERN and SLAC, precision measurements have tested the electroweak theory as a quantum field theory, at the one-per-mille level [5, 6, 6]. Last year, our colleagues working at LEP made a heroic push to discover the Higgs boson [8]. The search will intensify again in a few years at the Tevatron and the Large Hadron Collider.

The quark model of hadron structure and the parton model of hard-scattering processes have such pervasive influence on the way we conceptualize particle

\footnotetext{
${ }^{1} \mathrm{~A}$ recent article in FermiNews $[2$ makes the challenge uncomfortably plain.

${ }^{2}$ For surveys of the electroweak theory, with references, see Ref. [3, 4 .
} 
physics that quantum chromodynamics, the theory of strong interactions that underlies both, sometimes is taken for granted. QCD is a remarkably simple, successful [9], and rich theory of the strong interactions 10. The perturbative regime of QCD exists, thanks to the crucial property of asymptotic freedom, and describes many phenomena in quantitative detail. The strong-coupling regime controls hadron structure and gives us our best information about quark masses. Unfamiliar régimes of high density and high temperature contain riches we have only begun to explore.

The physics curriculum in the 1898-99 University of Chicago catalogue begins with a very triumphalist Victorian preface [11]:

"While it is never safe to affirm that the future of the Physical Sciences has no marvels in store even more astonishing than those of the past, it seems probable that most of the grand underlying principles have been firmly established and that further advances are to be sought chiefly in the rigorous application of these principles to all the phenomena which come under our notice .... An eminent physicist has remarked that the future truths of Physical Science are to be looked for in the sixth place of decimals."

As the ink was drying on these earnest words, Röntgen discovered $\mathrm{x}$ rays and published the epoch-making radiograph of his wife's hand, Becquerel and the Curies explored radioactivity, Thomson discovered the electron and showed that the "uncuttable" atom had parts, and Planck noted that anomalies in the first place of the decimals required a wholesale revision of the physicist's conception of the laws of Nature.

We have the benefit of a century of additional experience and insight, but we are not nearly so confident as our illustrious Victorian ancestors were that we have uncovered "most of the grand underlying principles." Indeed, while we celebrate the insights codified in the standard model of particle physics and look forward to resolving its puzzles, we are increasingly conscious of how little of the physical universe we have experienced and explored. Future truths are still to be found in precision measurements, but the century we are leaving has repeatedly shown that Nature's marvels are not limited by our imagination. Exploration can yield surprises that completely change what we think about - and how we think.

\subsection{Precision Measurements of Electroweak Observables}

The beautiful agreement between the electroweak theory and a vast array of data from neutrino interactions, hadron collisions, and electron-positron annihilations at the $Z^{0}$ pole and beyond means that electroweak studies have become a modern arena in which we can look for new physics "in the sixth place of decimals." Classic achievements include determining of the number of light neutrino species and inferring the mass of the top quark. [

The comparison between the electroweak theory and a considerable universe of data is shown in Figure 11 [12], where the pull, or difference between the

\footnotetext{
${ }^{3}$ These are reviewed in $\S 4.1$ of my TASI 2000 Lectures, Ref. [4.
} 


\begin{tabular}{|c|c|c|c|}
\hline \multicolumn{4}{|c|}{ Winter 2002} \\
\hline & Measurement & Pull & $\begin{array}{c}\left(\mathrm{O}^{\text {meas }}-\mathrm{O}^{\mathrm{fit}}\right) / \sigma^{\text {meas }} \\
-3-2-1 \quad 0 \quad 1 / 23\end{array}$ \\
\hline$\overline{\Delta \alpha_{\text {had }}^{(5)}\left(m_{z}\right)}$ & $0.02761 \pm 0.00036$ & -.27 & 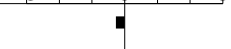 \\
\hline $\mathrm{m}_{\mathrm{z}}[\mathrm{GeV}]$ & $91.1875 \pm 0.0021$ & .01 & \\
\hline$\Gamma_{\mathrm{Z}}[\mathrm{GeV}]$ & $2.4952 \pm 0.0023$ & -.42 & 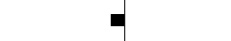 \\
\hline$\sigma_{\text {had }}^{0}[\mathrm{nb}]$ & $41.540 \pm 0.037$ & 1.63 & \\
\hline $\mathrm{R}_{1}$ & $20.767 \pm 0.025$ & 1.05 & L \\
\hline$A_{\mathrm{fb}}^{0,1}$ & $0.01714 \pm 0.00095$ & .70 & \\
\hline$A_{1}\left(P_{\tau}\right)$ & $0.1465 \pm 0.0033$ & -.53 & - \\
\hline $\mathrm{R}_{\mathrm{b}}$ & $0.21646 \pm 0.00065$ & 1.06 & 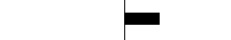 \\
\hline $\mathrm{R}_{\mathrm{c}}$ & $0.1719 \pm 0.0031$ & -.11 & , \\
\hline$A_{\mathrm{fb}}^{0, \mathrm{~b}}$ & $0.0994 \pm 0.0017$ & -2.64 & \\
\hline $\mathrm{A}_{\mathrm{fb}}^{0, \mathrm{c}}$ & $0.0707 \pm 0.0034$ & -1.05 & a \\
\hline$A_{b}$ & $0.922 \pm 0.020$ & -.64 & - \\
\hline$A_{c}$ & $0.670 \pm 0.026$ & .06 & \\
\hline$A_{1}(S L D)$ & $0.1513 \pm 0.0021$ & 1.50 & \\
\hline $\sin ^{2} \theta_{\text {eff }}^{\text {lept }}\left(Q_{\mathrm{fb}}\right)$ & $0.2324 \pm 0.0012$ & .86 & - \\
\hline $\mathrm{m}_{\mathrm{w}}[\mathrm{GeV}]$ & $80.451 \pm 0.033$ & 1.73 & \\
\hline$\Gamma_{\mathrm{W}}[\mathrm{GeV}]$ & $2.134 \pm 0.069$ & .59 & ש \\
\hline$m_{t}[G e V]$ & $174.3 \pm 5.1$ & -.08 & \\
\hline $\sin ^{2} \theta_{w}(v N)$ & $0.2277 \pm 0.0016$ & 3.00 & \\
\hline $\mathrm{Q}_{\mathrm{w}}(\mathrm{Cs})$ & $-72.39 \pm 0.59$ & .84 & 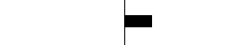 \\
\hline
\end{tabular}

Fig. 1. Precision electroweak measurements and the pulls they exert on a global fit to the standard model, from Ref. [12].

global fit and measured value in units of standard deviations, is shown for some twenty observables. The distribution of pulls for this fit, due to the LEP Electroweak Working Group, is not noticeably different from a normal distribution, and only a couple of observables differ from the fit by as much as two standard deviations. Whether the outliers $\left(A_{\mathrm{fb}}^{0, b}\right.$ and the NuTeV determination 13. of $\left.\sin ^{2} \theta_{\mathrm{W}}(\nu N)\right)$ contribute much-needed $\chi^{2}$ or point to new physics remains an open question [14].

\subsection{Electroweak Symmetry Breaking and Masses}

Elucidating the mechanism of electroweak symmetry breaking is the most urgent problem in particle physics, and the centerpiece of the LHC science program. The exploration of the $1-\mathrm{TeV}$ scale that will be needed to get to the bottom of electroweak symmetry breaking is frequently abbreviated as the search for the Higgs boson. That's a convenient sound bite, but we mustn't let the shorthand narrow our view of the task - and opportunity - we face. "The search for the Higgs boson" also fails to convey the full majesty of what we hope we will learn: why this world of stable structures - of diversity and change - is as we observe it 
to be, and not a formless fog of interchangeable parts flying about at the speed of light.

Another sound bite is even more pernicious because it is false: that is the bald assertion that the Higgs boson is the source of all mass. It is not. There are, in fact, several kinds of mass - so several sources of mass - and we have many interesting stories to tell.

The masses of the hadrons are (in principle, and with increasing precision in practice) understood from QCD in terms of the energy stored to confine a colorsinglet configuration of quarks in a small volume [15, 16. To say it another way: quantum chromodynamics explains nearly all of the visible mass in the universe. This is a remarkable achievement, and we should not be hiding it.

The Higgs boson is an essential part of the analogy to the Meissner effect in superconductivity that leads us to an excellent understanding of the masses of the electroweak gauge bosons $W^{ \pm}$and $Z^{0}$ as consequences of electroweak symmetry breaking. At tree level in the electroweak theory, we have

$$
\begin{aligned}
M_{W}^{2} & =g^{2} v^{2} / 2=\pi \alpha / G_{F} \sqrt{2} \sin ^{2} \theta_{W}, \\
M_{Z}^{2} & =M_{W}^{2} / \cos ^{2} \theta_{W},
\end{aligned}
$$

where the electroweak scale $v=\left(G_{F} \sqrt{2}\right)^{-\frac{1}{2}} \approx 246 \mathrm{GeV}$ is set by the vacuum expectation value of the Higgs field.

Interactions with the Higgs field may also generate quark and (charged) lepton masses; however, our understanding of these fermion masses is considerably more primitive. For each fermion mass, we require not just the scale of electroweak symmetry breaking, but a distinct and apparently arbitrary Higgsfermion-antifermion Yukawa coupling to reproduce the observed mass. In the electroweak theory, the value of each quark or charged-lepton mass is set by a new, unknown, Yukawa coupling. Taking the electron as a prototype, we define the left-handed doublet and right-handed singlet

$$
\mathrm{L}=\left(\begin{array}{c}
\nu_{e} \\
e
\end{array}\right)_{L}, \quad \mathrm{R} \equiv e_{R}
$$

Then the Yukawa term in the electroweak Lagrangian is

$$
\mathcal{L}_{\text {Yukawa }}^{(e)}=-\zeta_{e}\left[\overline{\mathrm{R}}\left(\varphi^{\dagger} \mathrm{L}\right)+(\overline{\mathrm{L}} \varphi) \mathrm{R}\right]
$$

where $\varphi$ is the Higgs field, so that the electron mass is $m_{e}=\zeta_{e} v / \sqrt{2}$. 5 For neutrinos, which may be their own antiparticles, there are still more possibilities for new physics to enter. Inasmuch as we do not know how to calculate the fermion Yukawa couplings $\zeta_{f}$, I believe that we should consider the sources of all fermion masses as physics beyond the standard model. It is even conceivable that fermion masses arise from some entirely distinct mechanism.

The values of the Yukawa couplings are vastly different for different fermions: for the top quark, $\zeta_{t} \approx 1$, for the electron $\zeta_{e} \approx 3 \times 10^{-6}$, and if the neutrinos have

\footnotetext{
${ }^{4}$ Although for the moment we take the weak mixing parameter $\sin ^{2} \theta_{W}$ from experiment, we understand how it arises in a unified theory. Moreover, in a unified theory we can hope to understand the parameter $\Lambda_{\mathrm{QCD}}$ that sets the scale of the hadron masses.

${ }^{5}$ Here I have taken the Yukawa coupling $\zeta_{e}$ to be real.
} 
Dirac masses, presumably $\zeta_{\nu} \approx 10^{-10}[$ What accounts for the range and values of the Yukawa couplings? Our best hope until now has been the suggestion from unified theories that the pattern of fermion masses simplifies on high scales. The classic intriguing prediction of the $S U(5)$ unified theory involves the masses of the $b$ quark and the $\tau$ lepton, which are degenerate at the unification point for a simple pattern of spontaneous symmetry breaking. The different running of the quark and lepton masses to low scales then leads to the prediction $m_{b} \approx 3 m_{\tau}$, in suggestive agreement with what we know from experiment [17].

Complex Yukawa couplings are a source of CP violation. Consider the piece of the Yukawa Lagrangian that gives masses to down-type quarks,

$$
-\mathcal{L}_{\text {Yukawa }}^{(d)}=\zeta_{i j}^{d}\left(\overline{\mathrm{L}}_{q_{i}} \varphi\right) \mathrm{R}_{d_{j}}+\zeta_{i j}^{d *} \overline{\mathrm{R}}_{d_{j}}\left(\varphi^{\dagger} \mathrm{L}_{d_{i}}\right)
$$

where the two terms are related by CP interchange. If the Yukawa coupling is not a real number, i.e., if $\zeta_{i j}^{d} \neq \zeta_{i j}^{d *}$, then $\mathrm{CP}$ is not a symmetry of $\mathcal{L}_{\text {Yukawa }}^{(d)}$. According to my convention that the sources of all fermion masses relate to physics beyond the standard model, the CP violation that we catalogue "within the standard model," which is to say arising from the quark mixing matrix (hence, the Yukawa couplings), is itself a window on physics beyond the standard model. Can we learn to read it for clues?

\section{$2.3 \nu$ Oscillation News}

The science that grew into particle physics began with found beams - the emanations from naturally occurring radioactive substance and the cosmic rays - and found beams still provide us with important windows on the universe. One of the great scientific detective stories of the recent past is the developing case for neutrino oscillations: the evidence that neutrinos produced as one flavor $\left(\nu_{e}, \nu_{\mu}\right.$, or $\nu_{\tau}$ ) actually morph into other flavors. Long known as a theoretical possibility, neutrino oscillation is now all but established by the Super-Kamiokande experiment's observation of an up-down asymmetry in the flux of muon neutrinos produced by the interaction of cosmic rays in the atmosphere [18]. By far the most graceful interpretation is that muon neutrinos produced on the far side of the Earth oscillate during flight in significant numbers into tau neutrinos.

The Sudbury Neutrino Observatory has added an important new element to our understanding of the longstanding puzzle of the solar neutrino deficit [19]. SNO reports an impressively precise measurement of the solar neutrino chargedcurrent cross section on the heavy-water that serves as their target-Cherenkov detector. The measured rate implies a $\nu_{e}$ flux

$$
\phi_{\mathrm{SNO}}^{\mathrm{CC}}\left(\nu_{e}\right)=1.75 \pm 0.07_{-0.11}^{+0.12} \pm 0.05 \times 10^{6} \mathrm{~cm}^{-2} \mathrm{~s}^{-1}
$$

where the uncertainties are statistical, systematic, and theoretical. They have also measured the solar neutrino elastic $\left(\nu_{x} e\right)$ cross section with limited precision, and extracted from it the flux of solar neutrinos of all active flavors,

$$
\phi_{\mathrm{SNO}}^{\mathrm{ES}}\left(\nu_{x}\right)=2.39 \pm 0.34_{-0.14}^{+0.16} \times 10^{6} \mathrm{~cm}^{-2} \mathrm{~s}^{-1} .
$$

${ }^{6} \mathrm{I}$ am quoting the values of the Yukawa couplings at a low scale typical of the masses themselves. 
The SNO experimenters are in the right place at the right time, because the Super-K experiment has already given a very precise measurement of the solar neutrino flux from elastic $\left(\nu_{x} e\right)$ scattering [20],

$$
\phi_{\mathrm{SK}}^{\mathrm{ES}}\left(\nu_{x}\right)=2.32 \pm 0.03_{-0.07}^{+0.08} \times 10^{6} \mathrm{~cm}^{-2} \mathrm{~s}^{-1} .
$$

The difference between the flux of active neutrinos and the flux of electron neutrinos,

$$
\phi_{\mathrm{SK}}^{\mathrm{ES}}\left(\nu_{x}\right)-\phi_{\mathrm{SNO}}^{\mathrm{CC}}\left(\nu_{e}\right)=0.57 \pm 0.17 \times 10^{6} \mathrm{~cm}^{-2} \mathrm{~s}^{-1},
$$

demonstrates at $3.3 \sigma$ that active neutrinos other than $\nu_{e}$, namely $\nu_{\mu}$ and $\nu_{\tau}$, arrive at Earth. Since the nuclear processes that power the Sun yield only $\nu_{e}$, this new result rules in favor of neutrino oscillations as the explanation for the solar neutrino puzzle.

\subsection{Clues about the Higgs-Boson Mass}

Of particular interest in the realm of electroweak measurements is the constraint on the mass of the Higgs boson. Figure 2 shows the $\Delta \chi^{2}$ curve derived from precision electroweak measurements performed at LEP — and by SLD, CDF, DØ, $\mathrm{NuTeV}$, and others - as a function of the Higgs-boson mass, within the standard model. The preferred value for the Higgs-boson mass, corresponding to the minimum of the curve, is around $85 \mathrm{GeV} / c^{2}$, with an experimental uncertainty of +54 and $-34 \mathrm{GeV} / c^{2}$ at $68 \%$ confidence level. This estimate is not materially affected by the NuTeV measurement discussed above.

Finding quantitative evidence that the virtual contributions of the Higgs boson are required to describe the observed constellation of electroweak data supports the theoretical contention that (something like) the Higgs boson must exist. IIt also directs our attention for the search into a narrower range than general theoretical arguments are capable of suggesting. The precision electroweak measurements tell us that the mass of the standard-model Higgs boson is lower than about $196 \mathrm{GeV} / \mathrm{c}^{2}$ (one-sided 95\% confidence level upper limit including both the experimental and the theoretical uncertainty). Since the direct searches at LEP [8] have concluded that $m_{H}>114.1 \mathrm{GeV} / c^{2}$, excluding much of the favored region, either the Higgs boson is just around the corner, or the standard-model analysis is misleading. One way or another, things will soon be popping!

\section{$3 \quad$ Big Questions}

In organizing my thoughts about the future agenda of particle physics, I find it useful to proceed from broad scientific themes to the specific questionsthe minute particulars - that will illuminate them, and then to instruments and

\footnotetext{
${ }^{7}$ Developed in $\S 5.1$ of my TASI 2000 lectures, Ref. 畉.
} 


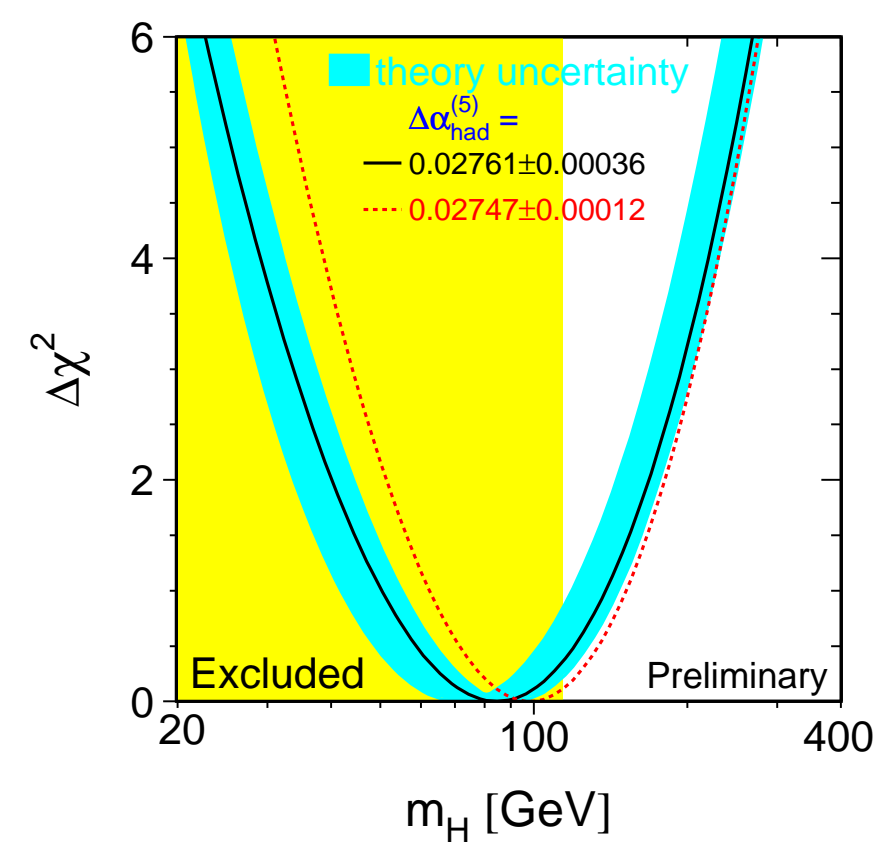

Fig. 2. $\Delta \chi^{2}=\chi^{2}-\chi_{\min }^{2}$ from a global fit to precision data vs. the Higgs-boson mass, $m_{H}$. The solid line is the result of the fit; the band represents an estimate of the theoretical error due to missing higher order corrections. The vertical band shows the $95 \% \mathrm{CL}$ exclusion limit on $m_{H}$ from the direct search at LEP. The dashed curve shows the sensitivity to a change in the evaluation of $\alpha\left(M_{Z}^{2}\right)$. (From the LEP Electroweak Working Group, Ref. [12.)

technology development. Here it will suffice to cite the main themes and develop them briefly.

Elementarity. Are the quarks and leptons structureless, or will we find that they are composite particles with internal structures that help us understand the properties of the individual quarks and leptons?

Symmetry. One of the most powerful lessons of the modern synthesis of particle physics is that (local) symmetries prescribe interactions. Our investigation of symmetry must address the question of which gauge symmetries exist (and, eventually, why). We have learned to seek symmetry in the laws of Nature, not necessarily in the consequences of those laws. Accordingly, we must understand how the symmetries are hidden from us in the world we inhabit. For the moment, the most urgent problem in particle physics is to complete our understanding of electroweak symmetry breaking by exploring the 1-TeV scale. With the passing of LEP2, this is the business of the experiments at the Tevatron Collider and 
the Large Hadron Collider.

Unity. In the sense of developing explanations that apply not to one individual phenomenon in isolation, but to many phenomena in common, unity is central to all of physics, and indeed to all of science. At this moment in particle physics, our quest for unity takes several forms.

First, we have the fascinating possibility of gauge coupling unification, the idea that all the interactions we encounter have a common origin and thus a common strength at suitably high energy.

Second, there is the imperative of anomaly freedom in the electroweak theory, which urges us to treat quarks and leptons together, not as completely independent species. Both of these ideas are embodied, of course, in unified theories of the strong, weak, and electromagnetic interactions, which imply the existence of still other forces - to complete the grander gauge group of the unified theoryincluding interactions that change quarks into leptons.

The third aspect of unity is the idea that the traditional distinction between force particles and constituents might give way to a unified understanding of all the particles. The gluons of QCD carry color charge, so we can imagine quarkless hadronic matter in the form of glueballs. Beyond that breaking down of the wall between messengers and constituents, supersymmetry relates fermions and bosons.

Finally, we desire a reconciliation between the pervasive outsider, gravity, and the forces that prevail in the quantum world of our everyday laboratory experience.

Identity. We do not understand the physics that sets quark masses and mixings. Although we are testing the idea that the phase in the quark-mixing matrix lies behind the observed $\mathrm{CP}$ violation, we do not know what determines that phase. The accumulating evidence for neutrino oscillations presents us with a new embodiment of these puzzles in the lepton sector. At bottom, the question of identity is very simple to state: What makes an electron and electron, and a top quark a top quark?

Topography. "What is the dimensionality of spacetime?" tests our preconceptions and unspoken assumptions. It is given immediacy by recent theoretical work. For its internal consistency, string theory requires an additional six or seven space dimensions, beyond the $3+1$ dimensions of everyday experience. Until recently it has been presumed that the extra dimensions must be compactified on the Planck scale, with a stupendously small compactification radius $R \simeq M_{\text {Planck }}^{-1}=$ $1.6 \times 10^{-35} \mathrm{~m}$. Part of the vision of string theory is that what goes on in even such tiny curled-up dimensions does affect the everyday world: excitations of the Calabi-Yau manifolds determine the fermion spectrum.

We have come to understand lately that Planck-length compactification is not - according to what we can establish-obligatory, and that current experiment and observation admit the possibility of dimensions not navigated by the strong, weak, and electromagnetic interactions that are almost palpably large. A whole range of new experiments will help us explore the fabric of space and time, in ways we didn't expect just a few years ago [21]. 


\section{The Role of Gravity}

\subsection{The Vacuum Energy Problem}

I want to spend a moment to revisit a longstanding, but usually unspoken, challenge to the completeness of the electroweak theory as we have defined it: the vacuum energy problem 22]. I do so not only for its intrinsic interest, but also to raise the question, "Which problems of completeness and consistency do we worry about at a given moment?" It is perfectly acceptable science - indeed, it is often essential - to put certain problems aside, in the expectation that we will return to them at the right moment. What is important is never to forget that the problems are there, even if we do not allow them to paralyze us.

For the usual Higgs potential, $V\left(\varphi^{\dagger} \varphi\right)=\mu^{2}\left(\varphi^{\dagger} \varphi\right)+|\lambda|\left(\varphi^{\dagger} \varphi\right)^{2}$, the value of the potential at the minimum is

$$
V\left(\left\langle\varphi^{\dagger} \varphi\right\rangle_{0}\right)=\frac{\mu^{2} v^{2}}{4}=-\frac{|\lambda| v^{4}}{4}<0
$$

Identifying $m_{H}^{2}=-2 \mu^{2}$, we see that the Higgs potential contributes a fieldindependent constant term,

$$
\varrho_{H} \equiv \frac{m_{H}^{2} v^{2}}{8}
$$

I have chosen the notation $\varrho_{H}$ because the constant term in the Lagrangian plays the role of a vacuum energy density. When we consider gravitation, adding a vacuum energy density $\varrho_{\text {vac }}$ is equivalent to adding a cosmological constant term to Einstein's equation. Although recent observations 8 raise the intriguing possibility that the cosmological constant may be different from zero, the essential observational fact is that the vacuum energy density must be very tiny indeed, 9

$$
\varrho_{\text {vac }} \lesssim 10^{-46} \mathrm{GeV}^{4} \text {. }
$$

Therein lies the puzzle: if we take $v=\left(G_{F} \sqrt{2}\right)^{-\frac{1}{2}} \approx 246 \mathrm{GeV}$ and insert the current experimental lower bound [8] $m_{H} \gtrsim 114 \mathrm{GeV} / c^{2}$ into (2), we find that the contribution of the Higgs field to the vacuum energy density is

$$
\varrho_{H} \gtrsim 10^{8} \mathrm{GeV}^{4}
$$

some 54 orders of magnitude larger than the upper bound inferred from the cosmological constant.

What are we to make of this mismatch? The fact that $\varrho_{H} \gg \varrho_{\text {vac }}$ means that the smallness of the cosmological constant needs to be explained. In a unified theory of the strong, weak, and electromagnetic interactions, other (heavy!) Higgs fields have nonzero vacuum expectation values that may give rise to still greater mismatches. At a fundamental level, we can therefore conclude that a spontaneously broken gauge theory of the strong, weak, and electromagnetic

\footnotetext{
${ }^{8}$ For a cogent summary of current knowledge of the cosmological parameters, including evidence for a cosmological constant, see Ref. 23.

${ }^{9}$ For a useful summary of gravitational theory, see the essay by T. d'Amour in $\S 14$ of the 2000 Review of Particle Physics, Ref. 24].
} 
interactions - or merely of the electroweak interactions - cannot be complete. Either we must find a separate principle to zero the vacuum energy density of the Higgs field, or we may suppose that a proper quantum theory of gravity, in combination with the other interactions, will resolve the puzzle of the cosmological constant. The vacuum energy problem must be an important clue. But to what?

\subsection{Neglecting Gravity}

It is entirely natural to neglect gravity in most particle-physics applications, because the coupling of a graviton $\mathcal{G}$ to a particle is tiny, generically of order $\left(E / M_{\text {Planck }}\right)$ where $E$ is a typical energy scale of the problem. Thus, for example, we expect the branching fraction $B(K \rightarrow \pi \mathcal{G}) \sim\left(M_{K} / M_{\text {Planck }}\right)^{2} \sim 10^{-38}$. And yet we cannot put gravity entirely out of our minds, even if we restrict our attention to standard-model interactions at attainable energies.

The great gap between the electroweak scale of about $10^{3} \mathrm{GeV}$ and the Planck scale of about $10^{19} \mathrm{GeV}$ gives rise to the hierarchy problem of the electroweak theory [25]: how to protect the Higgs-boson mass from quantum corrections that explore energies up to $M_{\text {Planck }}$. The conventional approach to the hierarchy problem has been to ask why the electroweak scale (and the mass of the Higgs boson) is so much smaller than the Planck scale. Framing the issue this way leads us to change the electroweak theory to include supersymmetry [26], or technicolor 22], or some other extension [28, 29]. Supersymmetry posits one or more new quantum dimensions, and entails a fermion-boson doubling of the particle spectrum. Superpartners on the $1-\mathrm{TeV}$ scale resolve the hierarchy problem by balancing the contributions of fermion and boson loops to the Higgs-boson mass renormalization. Models descended from technicolor ascribe the breaking of electroweak symmetry to a new strong interaction that becomes strong on the weak scale, making a composite Higgs boson. Many models developed over the past decade accord a privileged role to the top quark and third generation.

Over the past few years, we have begun instead to ask why gravity is so weak. This question motivates us to consider changing gravity to understand why the Planck scale is so large [30]. Now, elegant experiments that study details of Casimir and van der Waals forces tell us that gravitation closely follows

the Newtonian force law down to distances on the order of $0.3 \mathrm{~mm}$ [31], which corresponds to an energy scale of only about $10^{-12} \mathrm{GeV}$ ! At shorter distances (higher energies), the constraints on deviations from Newton's inverse-square force law deteriorate rapidly, so nothing prevents us from considering changes to gravity even on a small but macroscopic scale.

One way to change the force law is to imagine that gravity can propagate into extra dimensions. To respect the stronger constraints on the behavior of the standard-model interactions, we suppose that the $S U(3)_{c} \otimes S U(2)_{L} \otimes U(1)_{Y}$ gauge fields, plus needed extensions, reside on $(3+1)$-dimensional branes, not in the extra dimensions.

What difference do extra dimensions make? The dimensional analysis (Gauss's law, if you like) that relates Newton's constant to the Planck scale changes. If 
gravity propagates in $n$ extra dimensions with radius $R$, then

$$
G_{\text {Newton }} \sim M_{\text {Planck }}^{-2} \sim M^{\star-n-2} R^{-n},
$$

where $M^{\star}$ is gravity's true scale. Notice that if we boldly take $M^{\star}$ to be as small as $1 \mathrm{TeV} / \mathrm{c}^{2}$, then the radius of the extra dimensions is required to be smaller than about $1 \mathrm{~mm}$, for $n \geq 2$. If we use the four-dimensional force law to extrapolate the strength of gravity from low energies to high, we find that gravity becomes as strong as the other forces on the Planck scale. If the force law changes at an energy $1 / R$, as the large-extra-dimensions scenario suggests, then the forces are unified at lower energy $M^{\star}$. What we know as the Planck scale is then a mirage that results from a false extrapolation: treating gravity as four-dimensional down to arbitrarily small distances, when in fact - or at least in this particular fiction - gravity propagates in $3+n$ spatial dimensions. The Planck mass is an artifact, given by $M_{\text {Planck }}=M^{\star}\left(M^{\star} R\right)^{n / 2}$. If the true scale of gravity were close to $m_{H}$, the hierarchy problem would recede.

"Large" extra dimensions present us with new ways to think about the exponential variation of the Yukawa couplings that determine fermion masses. If the standard-model brane has a small thickness, the wave packets representing different fermion species might have different locations within the extra dimension [32]. On this picture, the Yukawa couplings measure the overlap in the extra dimensions of the left-handed and right-handed fermion wave packets and the Higgs field, presumed pervasive. Exponentially large differences might then arise from small offsets in the new coordinate(s). True or not, it is a mind-expanding way to look at an important problem.

\section{Concluding Remarks}

In the midst of a revolution in our conception of Nature, we confront many fundamental questions about our world of diversity and change. Are the quarks and leptons elementary or composite? What are the symmetries of Nature, and how are they hidden from us? Will we find new forms of matter, like the superpartners suggested by supersymmetry? Will we find additional fundamental forces? What makes an electron an electron and a top quark a top quark? What is the dimensionality of spacetime, what is its shape?

These are themselves great questions and, in the usual way of science, answering them can lead us toward the answers to yet broader and more cosmic questions. I believe that we are on the threshold of a remarkable flowering of experimental particle physics, and of theoretical physics that engages with experiment. Over the next decade or two, we may hope to

Understand electroweak symmetry breaking, Observe the Higgs boson, Measure neutrino masses and mixings, Establish Majorana neutrinos through the observation of neutrinoless double-beta decay, Thoroughly explore $\mathrm{CP}$ violation in $B$ decays, Exploit rare decays $(K, D, \ldots)$, Observe the neutron's permanent electric dipole meoment, and pursue the electron's electric dipole moment, Use top as a tool, Observe new phases of matter, Understand hadron structure 
quantitatively, Uncover the full implications of QCD, Observe proton decay, Understand the baryon excess of the universe, Catalogue the matter and energy of the universe, Measure the equation of state of the dark energy, Search for new macroscopic forces, Determine the gauge symmetry that unifies the strong, weak, and electromagnetic interactions, Detect neutrinos from the universe, Learn how to quantize gravity, Learn why empty space is nearly weightless, Test the inflation hypothesis, Understand discrete symmetry violation, Resolve the hierarchy problem, Discover new gauge forces, Directly detect dark-matter particles, Explore extra spatial dimensions, Understand the origin of the large-scale structure of the universe, Observe gravitational radiation, Solve the strong CP problem, Learn whether supersymmetry operates on the TeV scale, Seek TeV-scale dynamical symmetry breaking, Search for new strong dynamics, Explain the highest-energy cosmic rays, Formulate the problem of identity, ...

... and learn the right questions to ask 10

As we contemplate the interplay between experiment and theory that will lead us to a new, far-reaching understanding, it is inspiring to remember the words Michael Faraday recorded in his Research Notes of 19th March 1849:

"Nothing is too wonderful to be true,

if it be consistent with the laws of nature...

Experiment is the best test..."

Before closing, I'd like to step back from the specific aspirations of particle physics to recall the larger significance - the significance for human society - of what we do. In his millennial column in the New York Times, Anthony Lewis writes [33],

"[T] here has been one transforming change over this thousand years. It is the adoption of the scientic method: the commitment to experiment, to test every hypothesis. But it is broader than science. It is the open mind, the willingness in all aspects of life to consider possibilities other than the received truth. It is openness to reason."

Niels Bohr characterized the goal of science as the gradual reduction of prejudice. When we are at our best - when we are truest to our ideals - this spirit is what we offer to science, and to humanity.

What will we find at the LHC? Here is Cecil Frank Powell, recalling the wonders revealed in the first emulsions exposed on the Pic du Midi:

"It was as if, suddenly, we had broken into a walled orchard, where protected trees had flourished and all kinds of exotic fruits had ripened in great profusion."

\footnotetext{
${ }^{10}$ The vigorous discussion following my talk raised a number of other fascinating issues that will surely be much on our minds - whether we have the means to answer them soon, or not!
} 
Visions: The Coming Revolutions in Particle Physics

I expect wonders no less astonishing in the first collisions from the LHC. I look forward, with you, to breaking into a new walled orchard and feasting our eyesand our minds - on the exotic fruits we will find there.

\section{Acknowledgment}

It is a great pleasure to thank our hosts and organizers for the stimulating program and excellent welcome in Sardinia. I'd especially like to thank Gino Saitta and Carlo Bosio for their many contributions to the success of the workshop. I'm also grateful to Roger Cashmore for CERN's generous support of my participation.

\section{References}

1. Dava Sobel, Galileo's daughter : a historical memoir of science, faith, and love (Walker \& Co., New York, 1999).

2. Judy Jackson, "Interactions: Communicating particle physics in the twentyfirst century," FermiNews 25 (March 29, 2002) p. 6, available online at http://www.fnal.gov/pub/ferminews/ferminews02-03-29/p2.htm.

3. C. Quigg, Acta Phys. Polon. B30 (1999) 2145, hep-ph/9905369.

4. Chris Quigg, "The Electroweak Theory," in Flavor Physics for the Millennium, Proceedings of TASI 2000, edited by Jonathan L. Rosner (World Scientific, Singapore, 2001), pp. 3-67, FERMILAB-CONF-01-001-T, http://fnalpubs.fnal.gov/archive/2001/conf/Conf-01-001-T.htm.

5. A. Sirlin, "Ten Years of Precision Electroweak Physics," in Proc. 19th Intl. Symp. on Photon and Lepton Interactions at High Energy LP99, ed. J. A. Jaros and M. E. Peskin, Int. J. Mod. Phys. A 15S1 (2000) 398 [eConf C990809, 398 (2000)], hepph/9912227, http://www.slac.stanford.edu/econf/C990809/docs/sirlin.pdf.

6. M. Swartz, "Precision Electroweak Physics at the Z," in Proc. 19th Intl. Symp. on Photon and Lepton Interactions at High Energy LP99, ed. J. A. Jaros and M. E. Peskin, Int. J. Mod. Phys. A 15S1 (2000) 307 [eConf C990809, 307 (2000)], hepex/9912026, http://www.slac.stanford.edu/econf/C990809/docs/swartz.pdf.

7. D. Charlton, "Experimental tests of the standard model," to appear in the proceedings of International Europhysics Conference on High-Energy Physics (HEP 2001), Budapest, Hungary, 12-18 Jul 2001, hep-ex/0110086.

8. ALEPH Collaboration, DELPHI Collaboration, L3 Collaboration, OPAL Collaboration, the LEP Higgs Working Group, "Search for the Standard Model Higgs Boson at LEP," hep-ex/0107029.

9. C. Quigg, "The State of the Standard Model," in Physics Potential and Development of Muon Colliders and Neutrino Factories, edited by David B. Cline, AIP Conference Proceedings 542 (American Institute of Physics, Melville, NY, 2000), pp. 3-28, hep-ph/0001145.

10. F. Wilczek, Nucl. Phys. A663 (2000) 3.

11. S. B. Treiman, The Odd Quantum (Princeton University Press, Princeton, 1999).

12. LEP Electroweak Working Group, http://www.cern.ch/LEPEWWG/.

13. G. P. Zeller, et al. [NuTeV Collaboration], Phys. Rev. Lett. 88 (2002) 091802.

14. M. S. Chanowitz, Phys. Rev. Lett. 87 (2001) 231802, argues that, combining precision measurements and the Higgs boson search limit, the electroweak data favor new physics whether the $A_{\mathrm{fb}}^{0, b}$ anomaly is genuine or not. 
15. F. Wilczek, Phys. Today 52 (November, 1999) 11.

16. S. Aoki, et al. (CP-PACS Collaboration), Phys. Rev. Lett. 84 (2000) 238.

17. A. J. Buras, J. R. Ellis, M. K. Gaillard, and D. V. Nanopoulos, Nucl. Phys. B135 (1978) 66.

18. T. Toshito [Super-Kamiokande Collaboration], "Super-Kamiokande atmospheric $\nu$ results," hep-ex/0105023.

19. Q. R. Ahmad, et al. [SNO Collaboration], Phys. Rev. Lett. 87 (2001) 071301.

20. S. Fukuda, et al. [Super-Kamiokande Collaboration], Phys. Rev. Lett. 86 (2001) 5651.

21. J. Hewett and M. Spiropulu, "Particle-Physics Probes of Extra Spacetime Dimensions," to appear in Annu. Rev. Nucl. Part. Sci. 52 (2002).

22. M. Veltman, Phys. Rev. Lett. 34 (1975) 777; A. D. Linde, JETP Lett. 19 (1974) 183; J. Dreitlein, Phys. Rev. Lett. 33 (1977) 1243; S. Weinberg, Rev. Mod. Phys. 61 (1989) 1.

23. M. S. Turner, "Cosmological Parameters," in COSMO-98: Second International Workshop on Particle Physics and the Early Universe, edited by David O. Caldwell, AIP Conference Proceedings 478 (American Institute of Physics, Woodbury, NY, 1999) Woodbury, N.Y., Amer. Inst. Phys., 1999), p. 113, astro-ph/9904051.

24. D. E. Groom, et al. (Particle Data Group), Euro. Phys. J. C 15 (2000) 1 and 2001 off-year partial update for the 2002 edition at http://pdg.lbl.gov/

25. E. Gildener, Phys. Rev. D14 (1976) 1667; S. Weinberg, Phys. Lett. 82B (1979) 387.

26. B. C. Allanach, et al., "The Snowmass points and slopes: Benchmarks for SUSY searches," hep-ph/0202233.

27. C. T. Hill and E. H. Simmons, "Strong dynamics and electroweak symmetry breaking," hep-ph/0203079.

28. J. G. Branson, D. Denegri, I. Hinchliffe, F. Gianotti, F. E. Paige and P. Sphicas [ATLAS Collaboration and CMS Collaboration], "High transverse momentum physics at the Large Hadron Collider," hep-ph/0110021.

29. M. Carena, D. W. Gerdes, H. E. Haber, A. S. Turcot and P. M. Zerwas, "Executive summary of the Snowmass 2001 working group (P1) electroweak symmetry breaking," hep-ph/0203229.

30. Among the seminal papers, see I. Antoniadis, Phys. Lett. B246 (1990) 377; J. D. Lykken, Phys. Rev. D54 (1996) 3693; N. Arkani-Hamed, S. Dimopoulos, and G. Dvali, Phys. Lett. B429 (1998) 263.

31. C. D. Hoyle, et al., Phys. Rev. Lett. 86 (2001) 1418.

32. N. Arkani-Hamed, and M. Schmaltz, Phys. Rev. D61 (2000) 033005; E. A. Mirabelli and M. Schmaltz, Phys. Rev. D61 (2000) 113011.

33. Anthony Lewis, "Abroad at Home: The Fault, Dear Brutus," New York Times, 31 December 1999. 